$$
\text { "kovacs" — 2008/4/9 — 10:37 — page 393 — \#1 }
$$

\title{
WMI2: Interactive mathematics on the web
}

\author{
ZoLTÁN KovÁCS
}

\begin{abstract}
After 5 years of experiments and feedback we decided to continue the software development on WebMathematics Interactive, a web-based e-learning tool, rewriting it from scratch. The demonstration version of WebMathematics Interactive 2 (WMI2) has been shown to the expert audience on the CADGME conference. In this article we summarize the development goals and results.
\end{abstract}

Key words and phrases: computer algebra systems, computer aided education, distance learning, free software, modularized knowledge, Maxima, gnuplot, PHP, $\mathrm{T}_{\mathrm{E}} \mathrm{X} / \mathrm{L}_{\mathrm{E}} \mathrm{X}$, Linux.

ZDM Subject Classification: U55, U75.

\section{Introduction}

In 2003 we wrote a first report about the start of the WMI project [18] at the University of Szeged. Now our second article shows that our continuous work of the last years has raised into an international consensus. E-learning experts and didactical advisors joined us to enhance the abilities of WMI1. The result is a much more valuable tool in self-assessment of the learning process in mathematics. Though, further effort is needed to fulfill all the requirements we planned to have a complete education tool.

In this article, after outlining the past of WMI1, in Section 2 we build up some expectations of the future versions of WMI. Section 3 shows the results of our work; finally Section 4 gives a full example of problem solving using the new user interface of WMI2.

Copyright (C) 2007 by University of Debrecen 


$$
\text { "kovacs" — 2008/4/9 — 10:37 — page 394 — \#2 }
$$

\subsection{The past}

The main goal of WMI1 was to make a complete e-learning system for students at the age of $14-21$, containing basic services for solving routine problems (through CAS evaluation) and offering thematic modules of tutorials of selected areas of mathematics.

Our goal was partially achieved. WMI1 became a world-wide used frontend in several universities in Hungary. At University of Szeged we have been using this interactive web application during the every day teaching practise. In addition, scientific feedback was also recorded from the University of Pécs, College of Dunaújváros and College of Nyíregyháza. A dozen of introductory articles mentioned WMI1 during the last years, including both scientific and non-scientific papers $([1],[3],[9])$. International feedbacks arrived and scientific contacts were established with Taiwanese, British, French and Spanish colleagues. Presentations about WMI1 in mathematics conferences during CAS sections in Spain ${ }^{1}$, Slovakia $^{2}$, Hungary ${ }^{34}$ and Taiwan ${ }^{5}$ also showed that web based mathematics has a still growing audience of experts in the world.

Due to the statistics, our local installation of WMI1 — which also serves users internationally — processed and cached more than 900.000 on-line mathematical problems or subproblems since May 2004. We also reached a quite high Google PageRank of 5/10 (competitor CAS frontend Axiom [2] has the same rank; Wiris [6] and SAGE [11] have 6/10 in September 2007).

\section{Expectations}

Web based services are considered the most vibrating and modern way of computer aided technologies. The Google boom, i.e. the rapid growth of a freely available quality search engine, shows that "usability" usually means "nearness".

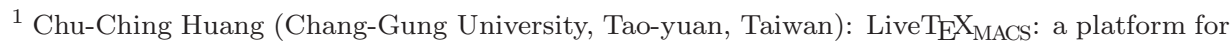
mathematical learning. International Congress of Mathematics 2006, Madrid

2 Michal Kaukič (University of Zilina, Slovakia): Possibilities and perspectives of open source software solutions for teaching and research at universities. Aplimat 2007, Bratislava

${ }^{3}$ Gábor Horváth, Antal Joós, Bálint Nagy (College of Dunaújváros, Hungary): WebMathematics Interactive in use. CADGME 2007, Pécs

${ }^{4}$ Zoltán Kovács: WebMathematics Interactive 2. Workshop, CADGME 2007, Pécs

${ }^{5}$ Chu-Ching Huang and Zoltán Kovács: Open resources for mathematical education. Mathematical meeting and annual meeting of the Mathemacial Society, Department of Mathematics, National Taiwan Normal University, 2006, Taipei
} 


$$
\text { "kovacs" — 2008/4/9 — 10:37 — page 395 — \#3 }
$$

If you find the information fast enough - without typing login user name and password - then you will come to the same information source next time, too, because you gained a positive experience. If not, probably you will not come any more. Timothy Cleamans, a young contributor of the robust SAGE project, as trying out WMI1, remarks: "What is interesting to me is how easy it is to use."

One of our main goal was always to focus on the ease of use. Much experience was collected during the years to enhance usability and make WMI more intuitive than before. In parallel, a second goal was to extend WMI to be a complete system to help in both self-learning and self-assessment.

During the last years many remarkable new software has appeared on the market and many among them have been freely available and useful. Indeed, a few goals of ours have been apparently implemented by other projects, some features of our implementation proved itself to be old fashioned, compared to the new kind of solutions. Our limited resources forced us to focus on such features of WMI1 which are still leading technologies world-wide, and we had to decide what kind of future developments are feasible for WMI at all. This is how we finally found out to concentrate on CAS based evaluation of routine problems and omit tutorial sections and questionnaires for some time. This means that WMI2 has less kind of built-in opportunities for its 2.0 release than for 1.0; hopefully for the next releases (2.1 and above) we shall focus on the missed features again.

From this point of view we listed all problems which could be solved within a limited time. In this section we summarize all such known problems of WMI1 and their planned solutions for WMI2.

\subsection{The student's view}

Problem 1 (InCOMPlete KNOWLEDGe BASE). There were too few areas of mathematics covered by WMI1. The basic services were limited to introductory calculus and algebra.

The source of this problem was the lack of technical knowledge of didactical designers. WMI1 was extremely difficult to expand due to the large amount of needed technical skills.

Problem 2 (Ambigous usage). The javascript based calculator was unable to start in Microsoft Internet Explorer and a wide range of users complained that it is not self-explanatory. 


$$
\text { "kovacs" — 2008/4/9 — 10:37 — page 396 — \#4 }
$$

Problem 3 (Circuitous work). The output formulas were not able to re-use in a following context. Many formulas had to be re-typed to continue the work with them in a similar, but not the same problem situation.

Problem 4 (Slowness). There was too much idle time between raising the question and getting the answer.

Problems 2, 3 and 4 could have been solved by skilled programmer technicians. Solving problem 2 seemed to be rather a didactical challenge than a programming skill.

\subsection{The teacher's view}

Problem 5 (Difficulties in extending). Problem 1 is very important from the teacher's view, too. To gain additional colleagues to encourage them to contribute their own ideas, exercises and solution methods, it is necessary to give an easy-to-use development tool for the teacher as well, preferably a web based editor.

Problem 6 (Math insertion). Entering math is a headache for most teachers. Both TEX and MathML (or simple HTML) seems to be infeasible to learn for elementary and grammar school teachers. Indeed, $\mathrm{T}_{\mathrm{E}} \mathrm{X}$ was designed for typesetting technicians, and MathML for mathematical web designers. A much more convenient way should be found for "mortal people".

Problem 7 (Customization). Flexible reorganization of the available learning material. Some teachers have their own logic of building up a theory or a part of mathematics. This demand should also be supported by web editor tools.

\subsection{Other enhancements}

A big project always have the danger that too much expectations should be fulfilled with limited resources. "Less is more", motto of minimalists, may make us understand that it can be more valuable to develop a tool which is useful for only one purpose but functioning perfectly, better than to have a multifunctional tool which is full of faults. In other words, if a well-known working tool already exists for a subproblem, then it should be checked if it is possible to merge with the existing system or not - if yes, that $3^{\text {rd }}$ party system should be used instead 


$$
\text { "kovacs" — 2008/4/9 — 10:37 — page 397 — \#5 }
$$

of starting or continuing to develop a brand new subsystem. The "only" work is just to create the communication interface between the 3rd party tool and the existing system.

After finishing WMI1 in May 2005 and patching its bugs until July 2006, it became clear for us to draw bright lines around the planning of the second version: what do we want to do for WMI2, and which features are more or less important. For that we set up a web server with a MediaWiki database and collected all ideas together: what to do and what to forget. This time interval for planning took almost 6 months. In December 2006 we started to build up the underlying database structure and fixate how WMI2 will work.

A very short version of the final decision of our judging committee follows:

Enhancement 1 (Problem database). A Google-like searching machinery is needed to classify mathematical problems. If it is possible, the classified problems must be able to be parameterized.

Enhancement 2 (Solution Charts). Thematic modules should be redesigned from scratch allowing teachers to design solution methods using flow charts.

Enhancement 3 (Assessment interface). Assessment techniques should co-operate with already working, existing, specialized systems.

\subsection{Summary of expectations}

During the kick-off of WMI1 development we defined the key properties for WMI. This has not been changed in our continous work. Here we summarize the longterm goals for the WMI development process:

- Be useful. WMI's intended audience is general level students from elementary school to graduate school (and their teachers). WMI should help make it easier to interactively experiment with mathematical objects. The content of the shown material should be as correct as possible in mathematical sense.

- Be efficient. WMI should be accessed via a web browser within a quick response time, even on slow workstations or low bandwith network (even on cellular phones). The expected technical knowledge of the user should not be more than general computer user skills. No programming or foreign language knowledge should be required: the software should be accessible in the mother tongue of the user. 


$$
\text { "kovacs" — 2008/4/9 — 10:37 — page 398 — \#6 }
$$

- Be free. There should always be a recent open source version of the software which is intended for public view. The components and underlying software of WMI should be open source software. There should always be at least one on-line website providing free access to WMI for anyone.

- Be cooperative. The software should reflect the Unix philosophy [20]: "Write programs that do one thing and do it well. Write programs to work together." This also implies that WMI should be well documented.

\section{The results}

In this section we summarize how Problems 1-7 and Enhancements 1-3 were solved in the development process.

During the implementation a clear picture was starting to be outlined that WMI2 should be a web based interface for freely available CAS and geometry software providing a textual worksheet containing the problems and their solutions in a savable and printable HTML format. Surprisingly, we found that other developer groups are also committed to similar goals. SAGE and Axiom have both a web interface for scientific problem solution and $\mathrm{T}_{\mathrm{E}} \mathrm{X}_{\mathrm{MACS}}[17]$, a native application for Unix-like operating systems, has also a very convenient interface to get on-the-fly answer from CAS, inside a user friendly environment. wxMaxima [16], a platform independent approach for such a system is also committed for international support of CAS usage.

The uniquity of WMI2 is that it focuses on education, not research or typesetting. It can be reached from everywhere on the internet independently of the used operating system and the web browser software. In addition, the intuitive graphical user interface is designed especially for young people at the age of 14-21 with its colorful theme and challenging layouts.

\subsection{Technical details}

To solve Problem 1 we created a database of 21 tables instead of the existing 10 ones in WMI1. All modifiable information is put down in a database. To enable web based editing, we used a commercial underlying database engine, Wekker, which is confirmed to be freely available for everyone to use. (The source code is not available.) Now all data is editable through a web interface, including creation of new calculator layouts which can cover different mathematical fields. 


$$
\text { "kovacs" — 2008/4/9 — 10:37 — page 399 — \#7 }
$$

We rewrote the javascript based calculator (Problem 2) to enable immediate check of the displayed result of a one-dimensional formula. We used bleeding edge Ajax technology [21] to connect formconv [12], IATEX, Maxima [14] and ImageMagick [15] to get a WYSIWYG formula editor, based on pure Javascript/DOM technology (no client-side Flash or Java applet used for portability, compatibility and speed reasons). These programming challenges shortly solved Problem 3 and 4, too.

Problem 5 was also solved using the Wekker Database Engine.

To solve Problem 6 we started to use the Xinha editor [4] which offers Javascript/MathML based on-the-fly formula editing. However, to harness the power of its equation editor, ASCIIMathML [10], the web browser should be carefully configured on a large variety of workstations. Usually a

To properly display the MathML on this page you need to install the following fonts...

message is sent within the browser, if configuration is not perfectly set up. That's why this problem cannot be considered to be solved completely. (The problem is that definition standard of the MathML language is still a work-in-progress.)

Problem 7 is also solved using the hierarchy editor of the Wekker Database Engine, however, latest version of WMI2 has no underlying database of a learning material tree yet.

Enhancements 1 and 2 are in planning stage yet. Enhancement 3 is expected to be solved via an on-line connection with Moodle [13].

\subsection{The next steps}

Finalizing WMI 2.0, we concluded the following plans for continuing the project:

- Detailed descriptions of the steps in the used methods during computing the solution. Theoretically we can offer arbitrary level of white box and black box content of such a step, as it is didactically desired. (See [5] for details.)

- Adding more basic services to cover all areas of mathematics, including linear algebra, numerical methods, number theory, Boole algebras. 


$$
\text { "kovacs" — 2008/4/9 — 10:37 — page } 400-\# 8
$$

The following table shows the differences between WMI1 and WMI2 and there is also a roadmap plan for versions $2.1^{6}, 2.2$ and 2.3. (2.0 has been released in September 2007.)

Table 1. Comparison of WMI1 and WMI2. (LAPP stands for Linux

+ Apache + PostgreSQL + PHP, see [19] for details.)

\begin{tabular}{|c|c|c|}
\hline Feature & WMI1 & WMI2 \\
\hline Basic services & 10 & $30(50+$ planned for 2.1$)$ \\
\hline - Calculator layouts & 3 & $5(8$ planned for 2.1$)$ \\
\hline - Re-usable input & Partial & Full \\
\hline - Re-usable output & - & Full \\
\hline - Exportable worksheet & - & HTML \\
\hline - Formula editor & $1 \mathrm{D}$ & $2 \mathrm{D}$ \\
\hline - Function plotting types & 3 & $1(3$ planned for 2.1$)$ \\
\hline - Function plotting zoom & In & In/out/moving planned for 2.1 \\
\hline - HTTP communication & Pages GET/POST & Ajax \\
\hline $\begin{array}{l}\text { - Problem and solution } \\
\text { caching }\end{array}$ & Yes & Yes \\
\hline Thematic modules & 5 (with 20 submodules) & Moodle interface planned for 2.1 \\
\hline $\begin{array}{l}\text { Interactive code } \\
\text { programming }\end{array}$ & PHP/CAS/gettext & CAS/flow charts planned for 2.3 \\
\hline Tree based knowledge & Yes & Planned for 2.2 \\
\hline - Searching in internet DBs & - & Planned for 2.3 \\
\hline Database editing & Only in source files & Web based \\
\hline $\begin{array}{l}\text { Time for a new service to } \\
\text { define }\end{array}$ & 1 day & 10 minutes \\
\hline PHP code readability & Poor & Better (Good planned for 2.1 ) \\
\hline- Size of code & $800 \mathrm{kB}$ & $200 \mathrm{kB}$ \\
\hline- Size of DB & 10 tables $(100 \mathrm{kB})$ & $20+$ tables $(50 \mathrm{kB})$ \\
\hline Design & Conservative, pastel-like & Bold, uproarious \\
\hline Documentation & Partial & Better (Good planned for 2.1) \\
\hline - International support & 8 languages & 2 languages (EN, HU) \\
\hline Suggested $C P U$ & Intel PIII $1.5 \mathrm{GHz}$ & Intel Xeon $4 \times 2.4 \mathrm{GHz}$ \\
\hline $\begin{array}{l}\text { Underlying software over } \\
L A P P\end{array}$ & CAS/gnuplot/formconv/php-cli & Maxima/gnuplot/formconv \\
\hline Live $C D$ available & Yes & - \\
\hline Ease of installation & Difficult & Average \\
\hline $\begin{array}{l}\text { Web page (demo } \\
\text { installation) }\end{array}$ & http://matek.hu/1 & http://matek.hu \\
\hline License & GNU GPL & GNU GPL (without Wekker) \\
\hline Downloadable from & http://sf.net/projects/wmi & http://matek.hu/downloads \\
\hline
\end{tabular}

${ }^{6}$ WMI 2.1 has been released on 17 December 2007. It contains 70 basic services in 6 layouts and 2 function plotting types (including zooming in and out). PHP code readability is good and documentation is full now. Installation process is reported to be "easy" by test users. End users also reported a high demand of explanations of solutions. WMI 2.1.1 is planned to be released in January 2008, including Chinese, Slovakian, Czech, French, Italian, German and Spanish translation as well. 


$$
\text { "kovacs" — 2008/4/9 — 10:37 — page } 401 \text { — \#9 }
$$

The following figures show a typical web page of WMI1 and WMI2.

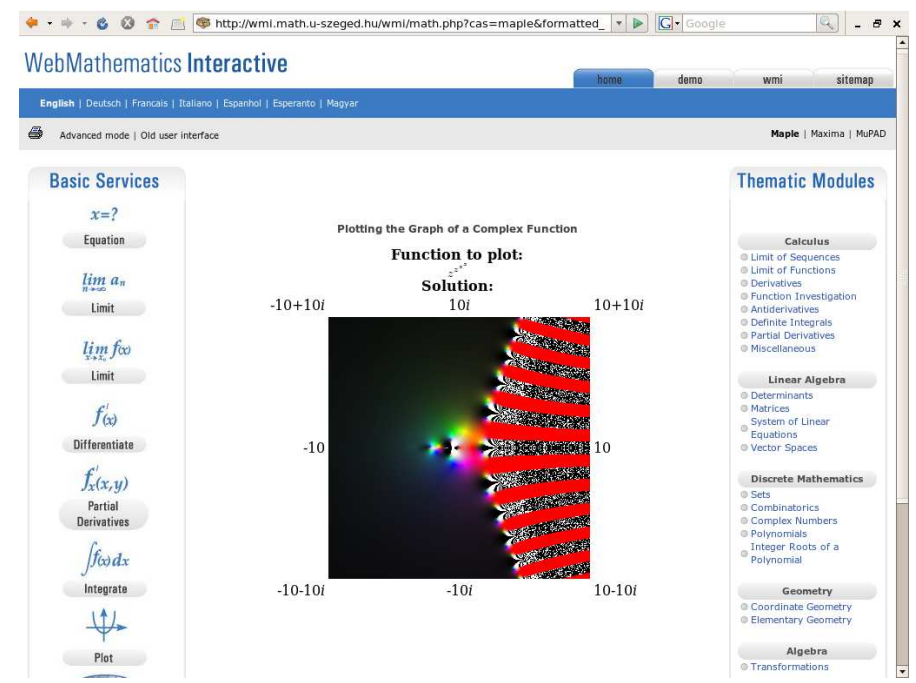

Figure 1. WMI1, plotting the complex function $f(z)=z^{z^{z}}$

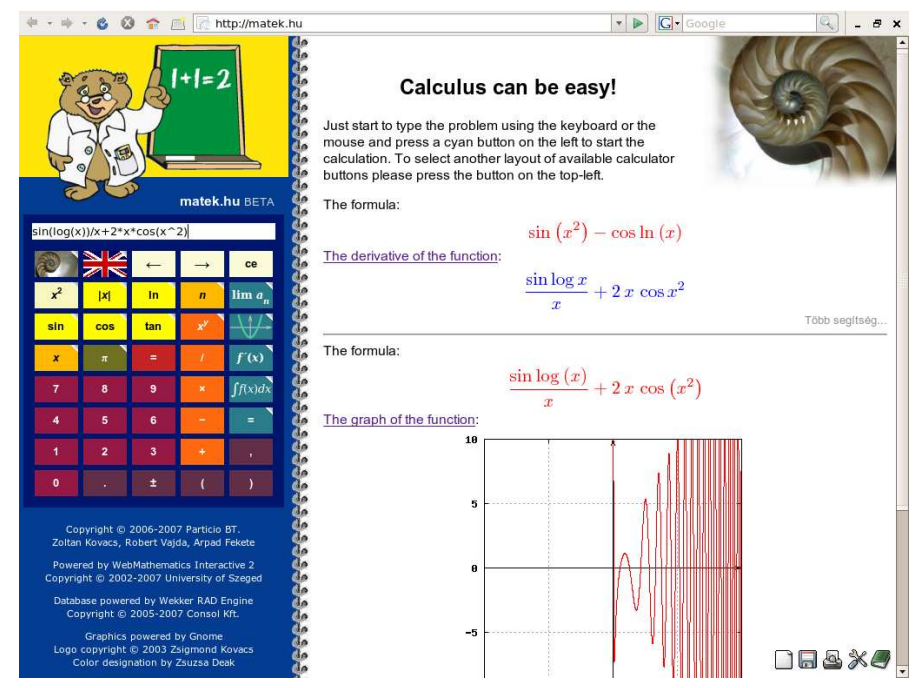

Figure 2. WMI2, calculus layout, calculating and plotting the derivative of the function $f: \mathbb{R}^{+} \rightarrow \mathbb{R}, f(x)=\sin x^{2}+\cos \ln x$ 


$$
\text { "kovacs" — 2008/4/9 — 10:37 — page } 402 \text { — \#10 }
$$

\section{How to use WMI2}

In this section we describe how WMI2 works from the user's point of view.

You need a recent web browser, Mozilla Firefox 2 is suggested, however Internet Explorer 6 and 7 also work properly. On the left side of the window of the web page there is a calculator which is designed for entering the formula of a problem, e.g. $x^{2}-\sin x$. The formula can be entered by typing it in a one dimensional "intuitive" form (i.e. $\mathrm{x}^{\wedge} 2-\sin (\mathrm{x})$ or $\mathrm{x}^{\wedge} 2$-sinx) or clicking on the following calculator buttons: $x, x^{2},--, \sin , x$. The two dimensional formula will be immediately shown on the right side of the window in red, displayed by the Ajax/PHP/formconv/LATEX/dvips/ImageMagick machinery.

Now we can work on this formula, applying one of the 30 currently available basic services:

- solving an equation, plotting a graph of a function;

- evaluation (or giving an approximated value) of an expression, simplification, factorization and expansion;

- limit of sequences and functions, differentiation, Taylor-series, antiderivatives, partial fractions, sums and products;

- dividing polynomials, greatest common divisor (gcd), least common multiplier $(\mathrm{lcm})$;

- matrix operations - including determinants, Gauss elimination (row reduced echelon form, triangulization), inverse, adjugate and adjoint matrix, eigenvalues and eigenvectors, characteristic polynomials, random matrices;

- binomial coefficients.

The CAS evaluation is powered by Maxima, plots are generated by Gnuplot [8]. The answer is shown in blue color. Both the question formula and the answer are put into a worksheet on the right side. Both formula can be re-used for further calculations by clicking on them. It is also allowed to use a former formula as a part of a new input. (The figure above shows an example of using WMI2 only by a mouse. The calculations illustrate the possibilities of WMI2 in an introductory calculus course.)

WMI2 also offers different calculator layouts for different type of students. Currently we offer 5 types of calculator layouts:

- Secondary school layout (default). It contains typical functions from secondary school mathematics (power, root, exponential, logarithmical, trigonometrical, signum, abs, floor and ceil) and binomial coefficients, gcd and lcm, 


$$
\text { "kovacs" — 2008/4/9 — 10:37 — page } 403 \text { — \#11 }
$$

factor and expand, evaluation, approximation and simplification; equations can be solved, and functions (in one variable) can be plotted.

- Elementary school layout. It offers power, floor, abs functions; solution of equations; function plotting; evaluation, approximation, simplification; gcd and $l \mathrm{~cm}$, factor and expand.

- Calculus layout. This gives the possibility to determine limits of sequences or functions, plotting them, differentiate or integrate and all appropriate CAS functions mentioned above.

- Algebra, polynomials. This layout is designed for solving discrete problems.

- Linear algebra. This layout is under construction, its purpose is to help calculating determinants, inverse of matrices, eigenvalues and similar routine problems.

Each layout has 5 buttons in 8 rows. These 40 buttons are usually not enough to list all available opportunities. To solve this problem, we put some similar types of buttons into the same button group, i.e. some buttons open up a set of new buttons available for further use.

\section{Acknowledgments}

Besides the author, the main concept of WMI was initiated by Róbert Vajda (University of Szeged). The biggest part of programming was made by Árpád Fekete (University of Szeged) as his master thesis [7] in computer science. Here we would like to thank our colleagues for their continuous encouragement and help, emphasizing Gábor Bakos (lead developer of formconv) and Kornél Csernai (technical assistant in PHP programming). In addition, our Taiwanese colleague, Chu-Ching Huang (Chang-Gung University, Tao-yuan, Taiwan) was also very kind to us in his full support during the development.

We also thank Christopher Sangwin (University of Birmingham), primary author of the STACK self-assessment system, for his worthy comments, including suggestion of considering Moodle as an interface for students (instead of using a native solution).

WMI2 was put together by dozens of mathematicians and programmers. We cannot give a full list of them here, but a complete enumeration of the contributors is available on http://wmi.sf net, primary page of WebMathematics Interactive. 


\section{References}

[1] T. Barik, $\mathrm{AT}_{\mathrm{E}} \mathrm{X}$ equations and graphics in PHP, Linux Journal (2005), http://www.linuxjournal.com/article/7870.

[2] M. Bronstein, W. Burge, T. Daly, J. Davenport, M. Dewar, M. Dunstan, A. Fortenbacher, P. Gianni, J. Grabmeier, J. Guidry, R. Jenks, L. Lambe, M. Monagan, S. Morrison, W. Sit, J. Steinbach, R. Sutor, B. Trager, S. Watt, J. Wen, and C. Williamson, The Axiom Book. The 30 Year Horizon, 2004, http://wiki.axiom-developer.org/public/book2.pdf.

[3] I. Blahota, Maxima: an open source CAS solution, Linuxvilág (September 2006), 36-39 (in Hungarian).

[4] M. Bazon, J. Sleeman, R. Meyer, et al., Xinha: The community-built open source online HTML editor, 2002-2007, http://xinha.webfactional.com.

[5] B. Buchberger, Should students learn integration rules?, ACM SIGSAM Bulletin 24, no. 1 (January 1990), 10-17.

[6] R. Eixarch, D. Marquès, and S. Xambó, Wiris, 1999-2007, http://www.wiris.com.

[7] Á. Fekete, WebMathematics Interactive 2, master thesis, University of Szeged, Department of Computer Science, 2007.

[8] H. P. Gavin, Gnuplot 4.0 - a brief manual and tutorial, 2000-2007, http://www.duke.edu/ hpgavin/gnuplot.html.

[9] J. Hubička, Z. Kovács, and Z. Kovács, Visualizations on the complex plane, Proceedings of Sprout-Selecting Conference, Pécs, Hungary, Computer Algebra Systems and Dynamic Geometry Systems in Mathematics Teaching (2004), 12-27.

[10] P. Jipsen, AsciiMathML, 2005, http://asciimathml.sourceforge.net/.

[11] D. Joyner, W. Stein, et al., SAGE Tutorial, 2004, http://www. sagemath.org/doc/html/tut/index.html.

[12] Z. Kovács and G. Bakos, Formula Converter, 2003-2007, http://formconv.sf .net.

[13] W. Rice, Moodle E-Learning Course Development, Packt Publishing, 2006.

[14] W. Schelter, J. Amundson, et al., Maxima Book, 1968-2007, http://maxima. sourceforge.net/docs/manual/en/maxima-5_13.pdf.

[15] S. Salehi, ImageMagick Tricks, Packt Publishing, 2006.

[16] SourceForge.net. wxMaxima, 2004-2007, http://wxmaxima.sf.net.

[17] J. van der Hoeven et al., $T_{E} X_{\text {MACS }}$, 1999-2007, http://www.texmacs.org.

[18] R. Vajda and Z. Kovács, Interactive web portals in mathematics, Teaching Mathematics and Computer Science 1, no. 2, Debrecen, Hungary (2003), 347-361.

[19] Wikipedia. LAMP (software bundle) - Wikipedia, the free encyclopedia, 2007, http://en.wikipedia.org/wiki/LAMP_(software_bundle).

[20] Wikipedia. Unix philosophy - Wikipedia, the free encyclopedia, 2007, http://en.wikipedia.org/w/index.php?title=Unix_philosophy.

[21] N. C. Zakas, J. McPeak, and J. Fawcett, Professional Ajax (Programmer to Programmer), Wrox, 2006. 
$\bigoplus$

$$
\text { "kovacs" — 2008/4/9 - 10:37 — page } 405-\# 13
$$

ZOLTÁN KOVÁCS

BOLYAI INSTITUTE

UNIVERSITY OF SZEGED

H-6701 SZEGED

ARADI VÉRTANÚK TERE 1.

PHONE: + 36-62-544647

HUNGARY

E-mail: kovzol@math.u-szeged.hu

(Received September, 2007) 